\title{
Relationship between Catecholamine and Renin- Angiotensin System in Spontaneously Hypertensive Rats
}

\author{
Nagao Kajiwara, Yoshiko Kobayashi, Jun Hashida, Takahisa \\ Uchiyama, Takashi Kita, Masahiko Satoh, Masashi Ono, \\ Yasuo Izeki, Tateo Tei, Hiroshi Terashima, Nobuaki \\ Mishina, Toshio Kushiro, and Michinobu Hatano
}

We have reported that catecholamine plays an important role in the pathogenesis of essential hypertension. Patients with essential hypertension sometimes show high concentration of both catecholamine and angiotension II in the blood, which may indicate an important relationship between these 2 pressor agents. The present study was designed to evaluate the interaction between plasma catecholamine and plasma renin activity (PRA) of SHR.

Materials and Methods:

Experimental animals consist of SHR (male, 6-month-old) and control Wistar rats (WR).

Three groups were prepared:

(1) Group of norepinephrine (NE) administration: Blood pressure (BP) and PRA were measured $30 \mathrm{~min}$ after intravenous infusion of $0.1 \mathrm{ml} / \mathrm{min}$ of NE solution ( $4 \mathrm{ng}$ in $5 \%$ glucose).

(2) Group of fusaric acid (FA) intraperitoneal administration: FA (100, 50,30 , and $20 \mathrm{mg} / \mathrm{Kg}$, respectively) was administered intraperitoneally and 3 hours later, blood pressure, PRA, and plasma NE were measured.

(3) Group of FA oral administration: FA $50 \mathrm{mg} / \mathrm{Kg} /$ day was administered orally for 7 days. Thereafter blood pressure and PRA were measured.

PRA was measured by bioassay and NE was by double isotope method.

Results and Discussion:

(1) After administration of NE, BP increased but PRA did not in WR, while in both BP and PRA increased in SHR.

(2) After intraperitoneal administration of FA, BP slightly decreased and PRA increased in WR, while in SHR BP significantly decreased and PRA increased. As to the blood pressure, the higher the blood pressure was, the more prominently it decreased and a dose-response relationship was recognized

From the Second Department of Internal Medicine, Nihon University School of Medicine and Department of Cardiology, Surugadai Nihon University Hospital, Tokyo. 
between the dose of FA and decrement of blood pressure. And NE showed tendency of decrease, when BP was lowered after administration of FA (Table).

Group of Intraperitoneal Administration of FA

\begin{tabular}{l|l|c|c|c}
\hline \multicolumn{2}{l|}{} & Syst BP $(\mathrm{mmHg})$ & PRA $(\mathrm{ng} / \mathrm{ml} / 18 \mathrm{hrs})$ & NE $(\mu \mathrm{g} / \mathrm{L})$ \\
\hline \multirow{3}{*}{ WR } & Control & $120-138$ & $20-26$ & \\
& FA $(30 \mathrm{mg} / \mathrm{Kg})$ & $102-110$ & $30-50$ & \\
\hline \multirow{5}{*}{ SHR } & Control & $170-182$ & $20-34$ & \\
& FA (100 mg/Kg) & $60-70$ & $30-40$ & \\
& Control & $162-200$ & $20-34$ & \\
& FA (50 mg/Kg) & $50-100$ & $34-58$ & \\
& Control & $170-182$ & $20-34$ & $0.6-1.5$ \\
& FA (30 mg/Kg) & $90-140$ & $52-60$ & $0.3-0.9$ \\
& Control & $190-194$ & $20-34$ & \\
& FA (20 mg/Kg) & $120-160$ & $26-46$ &
\end{tabular}

(3) BP significantly decreased but PRA did not change in SHR 7 days after oral administration of $F A(50 \mathrm{mg} / \mathrm{Kg} /$ day $)$.

After FA administration $(30 \mathrm{mg} / \mathrm{Kg})$ induced appropriate decrease in BP, PRA increased and NE decreased. Therefore, it seems that elevation of PRA was not due to increase of NE, but to the vasodilating effect of FA which causes hypotension and reduction of renal blood flow. After oral administration, BP decreased but PRA did not change significantly. This discrepancy could be due to the time needed to achieve proper BP and its final level.

\section{Summary:}

PRA and BP were measured after administration of NE and FA to evaluate the relationship between plasma NE and renin-angiotensin system in SHR.

(1) PRA increased after drip infusion of NE.

(2) BP decreased in proportion to the dose of FA administered and this effect was more prominent in the group with higher blood pressure before administration.

(3) PRA increased after intraperitoneal administration of FA, whose vasodilating effect could play a role as a renin releasing factor. During oral administration, BP decreased but PRA showed no change, and this discrepancy could be due to the time needed to achieve proper BP and its final level. 